\title{
The association value of random shapes revisited
}

RUSSELL EISENMAN

TEMPLE UNIVERSITY

Vanderplas \& Garvin (1959) found the mean association value of their random shapes to be only 38 per cent. Also, more Ss said "yes" than actually naming content. It was hypothesized that a more natural experimental procedure would lead to increased association value and increased content responses for the random shapes. Both hypotheses were supported beyond the .001 level.

Vanderplas \& Garvin (1959) seated Ss in a darkened experimental room and presented polygons for $3 \mathrm{sec}$. per polygon, the presentations being preceded and followed by buzzes. The Ss task was to report what association, if any, he could make to the polygon, or to say "yes"' if the shape reminded him of something which he was unable to name. The polygons were random shapes constructed according to a method suggested by Attneave \& Arnoult (1956). Vanderplas \& Garvin (1959) found a range of 20-to-62 percent as the association values for the various shapes, with a mean of 38 percent. Also, Ss more often said "yes" rather than giving content responses.

It is quite possible that being in a dark room, hearing buzzes, and having to perform verbally tended to decrease the association and content percentages. The present study was undertaken to see if Ss could give more association and content responses when writing their responses in the classroom, which is considered a more natural setting for college student Ss.

Subjects

The Ss were 22 students in a psychology class at Temple University. These Ss were tested as part of the class activity by their teacher.

\section{Stimulus Material}

Two photographs were employed: one of the Vanderplas \& Garvin (1959) shapes 5, 20, and 25 from the 4 point category; 1,12 , and 24 from the 12 point category; and, shapes 6,21 , and 24 from the 24 point category. This photograph showed the shapes as black figures on white ground, as Vanderplas \& Garvin (1959) had shown them. In addition, the photograph contained three symmetrical shapes taken from Birkhoff (1933). The Birkhoff shapes contained 4,8 , and 10 points. The second photograph was the same as the first except that the shapes were all photographed as white figures on black ground. The figure-ground reversals were obtained by use of a Docustat copying machine which routinely reverses the figure-ground relationship. Since no differences were obtained with the two different photographs, the data will be combined and figureground reversal considered an irrelevant issue in this study.

\section{Procedure}

As part of the class procedure the E provided each $S$ with a photograph of the polygons. The Ss were instructed to write down their association to each figure, or to mark "yes" if the figure reminded them of something which they were unable to name. Ss were also instructed to mark "no" if the figure did not remind them of anything. Theoretically, an unlimited time was made available to Ss, within the limitation of the $1 \mathrm{hr}$. 40 min. class period, to make their judgments. However, Ss were requested to mark down their first association and not to spend too much time on the polygons. The procedure was generally oriented toward making the experiment seem non-threatening and non-evaluative, in contrast to the hypothesized atmosphere of the Vanderplas \& Garvin (1959) study.

\section{Results}

For the Vanderplas \& Garvin (1959) shapes used in this study, association values in their study ranged from 28 to 50 percent, with a mean of 39 percent. For Ss in the present study, the range was 50 to 96 percent, with a mean of 67 percent. The difference between the Vanderplas \& Garvin (1959) results and the results from the present sample were assessed by the MannWhitney $U$ test, and were found to be highly significant $(U=1, p<.001)$.

Content responses, as opposed to "yes"' and "no" responses, ranged from 20 to 38 percent in the earlier study for the shapes employed here, with a mean of 29 percent. This is in marked contrast to the present study, in which the percentage of content responses ranged from 45 to 96 percent, with a mean of 63 percent. These non-overlapping distributions result in a MannWhitney $U$ of $0, p<.001$.

The Birkhoff (1933) symmetrical shapes had association values of 86,64 , and 100 percent for the 4,8 , and 10 point shapes, respectively. The content percentages for these shapes were 82,59 , and 82 percent, respectively. Thus, Ss tended to give more associations and more content responses to the Birkhoff (1933) symmetrical shapes than to the Vanderplas \& Garvin (1959) shapes.

\section{Discussion}

The results are consistent with the view that a more natural, relaxed experimental atmosphere can lead to different results than a situation in which the Ss are stressed, under evaluation, or otherwise having to perform in an atypical situation. While some kind of stress may be effective in emphasizing differences between groups, stressful procedure may be less valuable when the goal is to obtain norms, as in the Vanderplas \& Garvin (1959) research. 
Vanderplas \& Garvin (1959) found that simpler shapes allowed for more associations, a result which stands in contrast to that of Taylor \& Eisenman (1964). However, the latter study was dealing with art students, some of whom were highly creative. This $\mathrm{S}$ difference may be sufficient to account for the differences between the results of the two studies. The present data indicated that the 12-point polygons had more associations made to them. Two of the 12-point shapes had association values over 90 percent in the present study. Thus, complexity, as defined by the number of points, may not show as negative a relationship to association value as Vanderplas \& Garvin (1959) found. More intensive study seems needed before valid generalizations can be made.

If we think of the symmetrical shapes as simpler, then Vanderplas \& Garvin (1959) receive some support, in that more associations and content responses were made to the symmetrical Birkhoff (1933) shapes. It may be that the symmetry-asymmetry dimension is worthy of investigation in its own right, in addition to the complexity-simplicity dimension.

\section{References}

Attneave, F., \& Arnoult, M. D. Methodological considerations in the quantitative study of shape and pattern in perception. Psychol. Bull., 1956, 53, 452-471.

Birkhoff, G. D. Aesthetic measure. Cambridge: Harvard University Press, 1933.

Taylor, R. E., \& Eisenman, R. Perception and production of complexity by creative art students. J. Psychol., 1964, 57, 239-242.

Vanderplas, J. M., \& Garvin, E. A. The association value of random shapes. J. exp. Psychol., 1959, 57, 147-154. 